\title{
Double Image Segmentation Algorithm for Tick-Mark Recognition of Analog Instrument
}

\author{
Guidong Liu, Baoling Zhou, Wei Xie \\ Electronic and Information Engineering Department, Guangdong Baiyun University, Guangzhou, China \\ Email: public1976@163.com
}

How to cite this paper: Liu, G.D., Zhou, B.L. and Xie, W. (2021) Double Image Segmentation Algorithm for Tick-Mark Recognition of Analog Instrument. World Journal of Engineering and Technology, 9, 357373.

https://doi.org/10.4236/wjet.2021.92025

Received: April 7, 2021

Accepted: May 24, 2021

Published: May 27, 2021

Copyright $\odot 2021$ by author(s) and Scientific Research Publishing Inc. This work is licensed under the Creative Commons Attribution International License (CC BY 4.0).

http://creativecommons.org/licenses/by/4.0/ (c) (i) Open Access

\begin{abstract}
Tick-mark recognition is a key step for automatic reading of analog instrument with computer vision techniques which is called for in many automatic processes among industries. As many factors result in the tick-mark distortion in the sampled image such as the generally stained surface of instrument, or/and reflection and refraction of light, the accuracy and robustness of tick-mark reading are directly or indirectly weakened. Double image segmentation algorithm (DISA) is employed to solve this problem which makes coarse segmentation with structural parameters of instruments, and makes accurate segmentation with gray difference between tick-marks and their background. Tests have been conducted with $640 * 480$ pixel industry camera and 4 kinds of pointer pressure meter, and the results show that read-out rate of tick-mark in pointer meter reached $100 \%$ at sufficient illumination and $76 \%$ at insufficient illumination for air packed instrument. In addition with oil packed instrument, DISA still experienced $64 \%$ recognition in comparison with 0 success record by Hough Transformation.
\end{abstract}

\section{Keywords}

Machine Vision, Image Segmentation, Analog Instrument, Tick-Mark

\section{Introduction}

This template, created in MS Word 2007, provides authors with most of the formatting specifications needed for preparing electronic versions of their papers.

It is brought into reality with development of computer vision that analog instrument can be recognized with machine vision algorithm. This method could bring many advantages in analog instrument reading procedures including increased accuracy, shorted reading span, automatic data, remote transmission, avoiding boring repetitive working procedure and operator fatigue, and so on. 
As tick-mark recognition is a key step for automatic reading of analog instrument, it is now under intensive development by many researchers.

F Corrêa Alegria and A Cruz Serra [1] employed Hough Transform to get determine tick-marks. Chao Zheng et al. [2] proposed a robust and automatic recognition algorithm which can read the indication of analog instruments automatically at various brightness levels and camera angles. It is an integrated application of Multi-Scale Retinex with color restoration at different brightness levels, using Perspective Transform to get the front view of image taken from arbitrary camera angle, and using Hough Transform to determine the starting scale mark and ending scale mark.

Methodologically, tick-mark recognition can be divided two steps as follows:

1) Image Segmentation

Segmentation is a classical algorithm for image recognition mainly including threshold image segmentation [3] [4], region image segmentation [5] [6], edge image segmentation [7] [8] etc. As tick-marks have several properties as followings: symmetrically distributed on the edge of instruments, fixed distance between top of tick-marks and instrument center and fixed contrast ratio of tickmarks and their background, threshold image segmentation is relatively suitable for tick-mark recognition. In 1979 Otsu, N. [9] presented the famous Otsu threshold segmentation. This algorithm has been widely developed in different directions. For instance, Muthukumaran Malarvel et al. [10] addressed an improved version of Otsu's method which automatically selects a desired threshold value for image segmentation by adapting the Weibull distribution and the least non-zero probability value of gray-levels of the whole image has been considered as a weighted parameter of the background group of Otsu's within-class criterion. Ta Yang Goh et al. [11], based on Monte Carlo statistical method, shows that the success of image segmentation depends on object-background intensity difference, object size and noise measurement, however is unaffected by location of the object on that image.

2) Line Detection

As tick-mark takes on line characteristic, many algorithms could be employed to its detection including Hough transform. Kuo-Liang Chung et al. [12] presented an orientation-based DHT (ODHT) which consists of two strategies, the parameter space-selection strategy and the voting space-reduction strategy, to substantially reduce the computational complexity of the DHT. Besides its low computational merit, the proposed ODHT can also improve the detection accuracy of the DHT.

Yoshihiko Mochizuki et al. [13] introduced the N-point Hough transform for the detection of a large number of planar lines in a noisy image which guarantees the accuracy and robustness of line detection using the first and second sample points.

Theoretically, tick-marks could be detected with algorithms above on condition of clear images, but many inferior images could lead to deterioration of accuracy and robustness, even to recognition failure such as extreme brightness or 
darkness, difference between lens focus and instruments, noise arising from inferior cameras or dust panel, non-uniform illumination, and so on. Double image segmentation algorithm is employed to solve this problem which, on the one hand, coarsely positions the tick-mark searching pitch one by one depended on some structural parameters, and on the other hand, accurately picks up tick-mark from background depended on the estimated thresholds of tick-mark and background.

\section{Double Image Segmentation Algorithm (DISA)}

Purpose of tick-mark recognition of analog instrument is to position the tick marks in the coordinate system centered by instrument center for automatic recognition of analog instrument.

DISA algorithm is developed in recognition of pointer pressure meters used in railway locomotives, which three steps including preparation, coarse image segmentation and accurate image segmentation. In pretreatment, two characteristic parameters are recognized such as center and radius of the instrument panel, and in the rest steps, brightness barycenter of tick marks are searched one by one as an estimation of Geometric center tick marks with double image segmentation.

\section{Step 1. Preparation}

Observation of the analog instrument in Figure 1 can draw some conclusion about the tick-marks as following: 1) fixed distance between top of tick-marks and instrument center; 2) equal angle span between neighbor tick-marks centered on instrument center. These properties mean the precondition of tick-mark finding is obtainment of instrument center and the radius of the panel.

According to the observation, it is found that there is evident difference between the instrument panel and other area, especially background, and at the same time, the brightness distribution of the panel is uniform relatively. Thus the geometrical center of the instrument is estimated by the brightness barycenter of the panel as following.

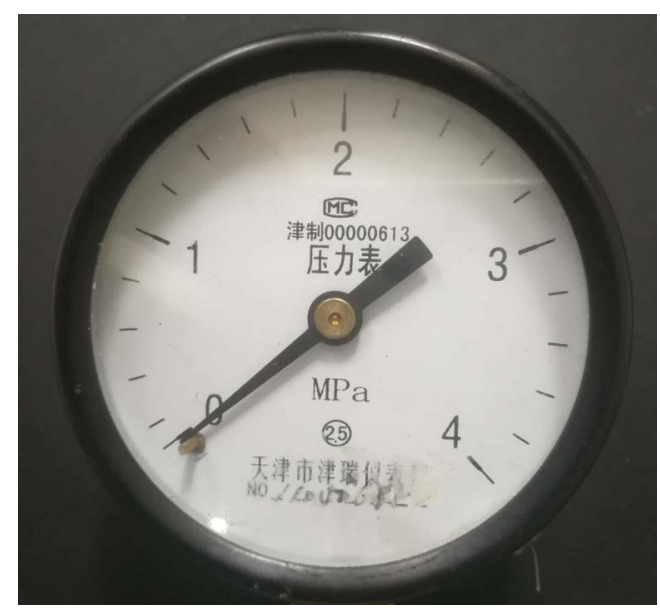

Figure 1. Panel of a pressure instrument. 


$$
\left\{\begin{array}{l}
x_{\text {instrument-cener }}=\sum_{i=0}^{L} \sum_{j=0}^{C} i B(i, j) \\
y_{\text {instrument-center }}=\sum_{i=0}^{L} \sum_{j=0}^{C} j B(i, j)
\end{array} \text { when } B(i, j)>T_{\text {background }}\right.
$$

where $i$ and $j$ are the line index and column index of pixel respectively, and $L, C$ are the maximum index of line and column; $B(i, j)$ is the gray value of pixel; $T_{\text {background }}$ is the grey threshold of all pixels in the image out of panel zone where grey value is bigger than any grey value of pixels in background; ( $\left.x_{\text {center }}, y_{\text {center }}\right)$ is the gray barycenter of panel in estimation of geometrical center of the panel.

In addition, the radius of the panel, $R_{\text {panel }}$, can be approximately computed as Formula (2):

$$
R_{\text {panel }}=\sqrt{\frac{N_{\text {panel }}}{\pi}}
$$

where $N_{\text {panel }}$ is the summation of all pixels whose grey values are bigger than $T_{\text {background }}$.

\section{Step 2. Coarse Image Segmentation}

Coarse image segmentation is the process of positioning the zone of the current tick mark, and dividing the tick mark searching pitch from the whole image according to characteristic parameters of instrument panel such as instrument center, tick mark center, distance between tick mark and instrument center and so on. The purpose of the operation is to reduce the negative effects of uneven illumination, attached dirt, or shadow on the instrument panel during tick mark positioning.

For recognition of tick-marks with iteration, coarse image segmentation is performed to obtain the 4 vertexes of the tick mark searching pitch for tick-mark described as follows:

$$
\left\{\begin{array}{l}
\left(x_{\text {current,min }}, y_{\text {current,min }}\right) \\
\left(x_{\text {current,min }}, y_{\text {current,max }}\right) \\
\left(x_{\text {current,max }}, y_{\text {current,min }}\right) \\
\left(x_{\text {current,max }}, y_{\text {current,max }}\right)
\end{array}\right.
$$

where, $x_{\text {current,min }}, x_{\text {current,max }}, y_{\text {current,min }}$ and $y_{\text {current,max }}$ are the border of the tick mark searching pitch which is separated from other image which include the message of the current tick-mark and its background.

If the current tick-mark is the first tick-mark, then

$$
\left\{\begin{array}{l}
x_{\text {current,min }}=\hat{x}_{0, \text { center }}-\hat{l}_{0, \text { tick-mark }} \\
y_{\text {current,min }}=\hat{y}_{0, \text { center }}-\frac{1}{2} \hat{l}_{0, \text { tick-mark }} \\
x_{\text {current,min }}=\hat{x}_{0, \text { center }}+\hat{l}_{0, \text { tick-mark }} \\
y_{\text {current,max }}=\hat{y}_{0, \text { center }}+\frac{1}{2} \hat{l}_{0, \text { tick-mark }}
\end{array}\right.
$$


else

$$
\left\{\begin{array}{l}
x_{\text {current,min }}=\hat{x}_{\text {current,center }}-\hat{l}_{\text {current,tick-mark }} \\
y_{\text {current,min }}=\hat{y}_{\text {current,center }}-\frac{1}{2} \hat{l}_{\text {current,tick-mark }} \\
x_{\text {current,min }}=\hat{x}_{\text {current,center }}+\hat{l}_{\text {current,tick-mark }} \\
y_{\text {current,max }}=\hat{y}_{\text {current,center }}+\frac{1}{2} \hat{l}_{\text {current,tick-mark }}
\end{array}\right.
$$

The physical meanings of these parameters are listed in Table 1.

These parameters are estimated as:

$$
\left\{\begin{array}{l}
\hat{l}_{0, \text { tick-mark }}=\frac{1}{10} R_{\text {panel }} \\
\hat{l}_{\text {current,tick-mark }}=l_{\text {former,tick-mark }} \\
\hat{x}_{0, \text { center }}=\frac{1}{2} \hat{l}_{0, \text { tick-mark }} \\
\hat{y}_{0, \text { center }}=y_{\text {instrument-center }} \\
\hat{x}_{\text {current,center }}=x_{\text {former,center }}+D_{\text {former }} \cos \left(\hat{\theta}_{\text {current,scale }}\right) \\
\hat{y}_{0, \text { center }}=y_{\text {former,center }}+D_{\text {former }} \sin \left(\hat{\theta}_{\text {current,scale }}\right) \\
\hat{\theta}_{0, \text { center }}=0 \\
\hat{\theta}_{\text {current,scale }}=\theta_{\text {former,center }}+\Delta \theta_{\text {former }}
\end{array}\right.
$$

The physical meanings of these parameters, $l_{\text {former, tick-mark }}$, $\left(x_{\text {former, center }}, y_{\text {former, center }}\right), D_{\text {former }}, \theta_{\text {former,scale }}$ and $\Delta \theta_{\text {former }}$, are listed in Table 2.

Table 1. The physical meanings of $\left(\hat{x}_{0, \text { center }}, \hat{y}_{0, \text { center }}\right), \hat{l}_{0, \text { tick-mark }},\left(x_{\text {current, center }}, y_{\text {current,center }}\right)$, and $l_{\text {current,tick-mark }}$.

\begin{tabular}{cc}
\hline symbol & meaning \\
\hline$\left(\hat{x}_{0, \text { center }}, \hat{y}_{0, \text { center }}\right)$ & estimation of the geometrical center for first tick-mark \\
$l_{0, \text { tick-mark }}$ & estimation of the tick-mark length for first tick-mark \\
$\left(x_{\text {current,center }}, y_{\text {current,center }}\right)$ & estimation of the geometrical center for non first tick-mark \\
$l_{\text {current,tick-mark }}$ & estimation of the tick-mark length for non first tick-mark
\end{tabular}

Table 2. The physical meanings of $l_{\text {former,tick-mark }},\left(x_{\text {former,center }}, y_{\text {former,center }}\right), D_{\text {former }}, \theta_{\text {former,scale }}$ and $\Delta \theta_{\text {former }}$.

\begin{tabular}{cl}
\hline symbol & meaning \\
\hline$l_{\text {former, tick-mark }}$ & tick-mark length of former tick-mark \\
$\left(x_{\text {former, center }}, y_{\text {former, center }}\right)$ & Brightness barycenter center of former tick-mark \\
$D_{\text {former }}$ & Distance between $\left(x_{\text {former, center }}, y_{\text {former, center }}\right)$ and $\left(x_{\text {center }}, y_{\text {center }}\right)$ \\
$\theta_{\text {former, scale }}$ & Angle of former tick mark on reference to coordinate origin $\left(x_{\text {center }}, y_{\text {center }}\right)$ \\
$\Delta \theta_{\text {former }}$ & Difference between $\theta_{\text {former, scale }}$ and its former tick mark
\end{tabular}




\section{Step 3. Accurate Image Segmentation}

Accurate image segmentation is the process of dividing tick mark pixels from the searching pitch according to $T_{\text {current, tick-mark }}$ and $T_{\text {current,background }}$, the brightness thresholds of the current tick mark and its background and at the same time, computing the following parameters for tick mark searching iteration in Table 3.

As brightness thresholds of tick-mark and its background, $T_{\text {current,tick-mark }}$ and $T_{\text {current,background }}$, can't be obtained until their pixels been searched arithmetically, these two parameters can been estimated as $\hat{T}_{\text {current,tick-mark }}$ and $\hat{T}_{\text {current,background }}$. If first tick mark is searched, $\hat{T}_{\text {current, tick-mark }}$, is approximately estimated with brightness average of pixels around the instrument center, and $\hat{T}_{\text {current,background }}$, can been regarded as the brightness average of the instrument panel. In other case, $\hat{T}_{\text {current, tick-mark }}$ and $\hat{T}_{\text {current, background }}$, can been computed as following.

$$
\begin{aligned}
\hat{T}_{\text {current, tick-mark }} & =G A_{\text {former, tick-mark }} \\
\hat{T}_{\text {current,background }} & =G A_{\text {former, background }}
\end{aligned}
$$

where $G A_{\text {former, tick-mark }}$ and $G A_{\text {former, background }}$ are the brightness average of the former tick mark and its background.

1) Pixel classification

Grey value of pixels, $G V_{\text {current }, x, y}$, in the searching pitch with vertex as $\left(x_{\text {current,min }}, y_{\text {current,min }}\right),\left(x_{\text {current,max }}, y_{\text {current,max }}\right)$ can been classified into two categories: tick-mark category $C L A S S_{\text {current, tick-mark }}$ and background category

CLASS $S_{\text {current,background }}$, according to $\hat{T}_{\text {current, tick-mark }}$ and $\hat{T}_{\text {current,background }}$ respectively. If the brightness of pixel $P_{\text {current }, x, y}$ satisfy Formula (9).

$$
\begin{aligned}
G V_{\text {current }, x, y}< & \frac{1}{2}\left(\hat{T}_{\text {current, tick-mark }}+\hat{T}_{\text {current, background }}\right) \\
& -\frac{1}{2} \alpha\left(\hat{T}_{\text {current, background }}-\hat{T}_{\text {current, tick-mark }}\right)
\end{aligned}
$$

Then

\begin{tabular}{|c|c|}
\hline symbol & meaning \\
\hline$\left(x_{\text {current, center }}, y_{\text {current,center }}\right)$ & brightness barycenter of tick mark \\
\hline$G A_{\text {current, tick-mark }}$ & brightness average of tick mark \\
\hline$G A_{\text {current,background }}$ & brightness average of tick mark's background \\
\hline$\theta_{\text {current, tick-mark }}$ & angle of $\left(x_{\text {current, center }}, y_{\text {current,center }}\right)$ reference to $\left(x_{\text {instrument-cener }}, y_{\text {instrument-center }}\right)$ \\
\hline$\Delta \theta_{\text {current }}$ & difference between $\theta_{\text {former,scale }}$ and its former tick mark \\
\hline$D_{\text {current }}$ & distance between $\left(x_{\text {current,center }}, y_{\text {current,center }}\right)$ and $\left(x_{\text {center }}, y_{\text {center }}\right)$ \\
\hline
\end{tabular}

$$
P_{\text {current }, x, y} \subset C L A S S_{\text {current, tick-mark }}
$$

Table 3. Parameters' computing for tick mark searching iteration. 
where, $x, y$ are the line index and column index respectively, and $\alpha$ is filtering factor of tick-mark.

And if the brightness of pixel $P_{\text {current }, x, y}$ satisfy Formula (11).

$$
\begin{aligned}
G V_{\text {current }, x, y}> & \frac{1}{2}\left(\hat{T}_{\text {current, tick-mark }}+\hat{T}_{\text {current, background }}\right) \\
& -\frac{1}{2} \beta\left(\hat{T}_{\text {current, background }}-\hat{T}_{\text {current, tick-mark }}\right)
\end{aligned}
$$

then

$$
P_{\text {current }, x, y} \subset C L A S S_{\text {current, background }}
$$

where, $\beta$ is the filtering factor for background searching.

2) Computing $\left(x_{\text {current,center }}, y_{\text {current,center }}\right)$, the barycenter of the pixels in CLASS $S_{\text {current, tick-mark }}$ and its brightness average, $G A_{\text {current, tick-mark }}$

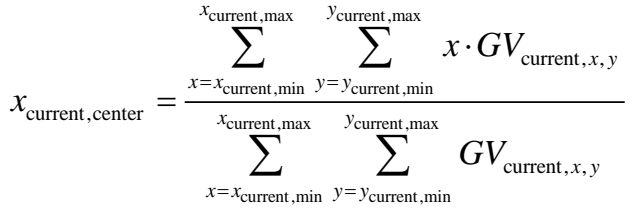

$$
\begin{aligned}
& y_{\text {current, center }}=\frac{\sum_{x=x_{\text {current, min }}}^{x_{\text {current,max }}} \sum_{x=y_{\text {current, min }}}^{x_{\text {current, min }}} \sum_{y=y_{\text {current, min }}}^{y_{\text {current,max }}} G \cdot G V_{\text {current }, x, y}}{y_{\text {current,max }}} \\
& G A_{\text {current, tick-mark }}=\frac{\sum_{x=x_{\text {current,min }}}^{x_{\text {current,max }}} \sum_{y_{\text {current, min }}}^{y_{\text {current,max }}} G V_{\text {current }, x, y}}{\left(x_{\text {current, max }}-x_{\text {current,min }}\right)\left(y_{\text {current,max }}-y_{\text {current,min }}\right)}
\end{aligned}
$$

where $P_{\text {current }, x, y} \subset C L A S S_{\text {current, tick-mark }}$.

3) Computing $G A_{\text {current,background }}$, the grey average of the pixels in CLASS $_{\text {current,background }}$ as following:

$$
G A_{\text {current, background }}=\frac{\sum_{x=x_{\text {current, min }}}^{x_{\text {current,max }}} \sum_{y_{\text {current, min }}}^{y_{\text {current,max }}} G V_{\text {current }, x, y}}{\left(x_{\text {current, max }}-x_{\text {current, min }}\right)\left(y_{\text {current,max }}-y_{\text {current,min }}\right)}
$$

4) Computing $\theta_{\text {current, tick-mark }}$, the angle of the center of the current tick-mark in the coordinate system centered with the instrument center as follows

$$
\begin{aligned}
& \theta_{\text {current, tick-mark }} \\
& =\left\{\begin{array}{l}
\frac{\pi}{2} \text { if } x_{\text {current,center }}=x_{\text {instrument-center }} \\
\pi+\tan ^{-1}\left(\frac{y_{\text {current, center }}-y_{\text {instrument-center }}}{x_{\text {current, center }}-x_{\text {instrument-center }}}\right) \text { if } x_{\text {current,center }}>x_{\text {instrument-center }} \\
\tan ^{-1}\left(\frac{y_{\text {current,center }}-y_{\text {instrument-center }}}{x_{\text {current,center }}-x_{\text {instrument-center }}}\right) \text { if } x_{\text {current, center }}<x_{\text {instrument-center }}
\end{array}\right.
\end{aligned}
$$

5) Computing $\Delta \theta_{\text {current }}$, the difference between $\theta_{\text {current, tick-mark }}$ and $\theta_{\text {former, tick-mark }}$. 


$$
\Delta \theta_{\text {current }}=\theta_{\text {current, tick-mark }}-\theta_{\text {former,tick-mark }}
$$

where, $\theta_{\text {former,tick-mark }}$ is the angle of the center of the former tick-mark in the coordinate system centered with the instrument center.

6) Computing $D_{\text {current }}$, the distance between the current tick mark and the instrument center.

$$
D_{\text {current }}=\sqrt{\left(x_{\text {current, center }}-x_{\text {instrument-center }}\right)^{2}+\left(y_{\text {current,center }}-y_{\text {instrument-center }}\right)^{2}}
$$

Cycling between step (2) and step (3), and adaptively the parameters above for every tick-mark, till all the tick marks are found.

\section{Results and Discussion}

The test bench in this paper was designed and assembled by GBU (Guangdong Baiyun University). This test bench, as shown in Figure 2, mainly includes the analog pressure instrument, camera, and a sliding table which has a function of distance adjusting between the instrument and the camera for focal length optimization. The parameters of the camera is as follows:

1) Effective Pixels: 640 * 480 ,

2) Pixel size: $2.2 * 2.2 \mu \mathrm{m}$,

3) Pixel bit depth: 12 bit,

4) Sensitivity: $1.76 \mathrm{~V} / \mathrm{lux}-\mathrm{sec} 550 \mathrm{~nm}$,

5) Sensor type: $1 / 2.5^{\prime \prime}$ CMOS.

The parameters of the analog pressure instrument is as follows:

1) Type: Bourdon tube,

2) Range: 0 - 1.6 MPa,

3) Diameter: $100 \mathrm{~mm}$,

4) Number of tick-marks: 51.

A typical panel of pressure instrument presented in Figure 3 shows that rectangular tick-marks are distributed on the edge of the instrument panel with following properties in the image.

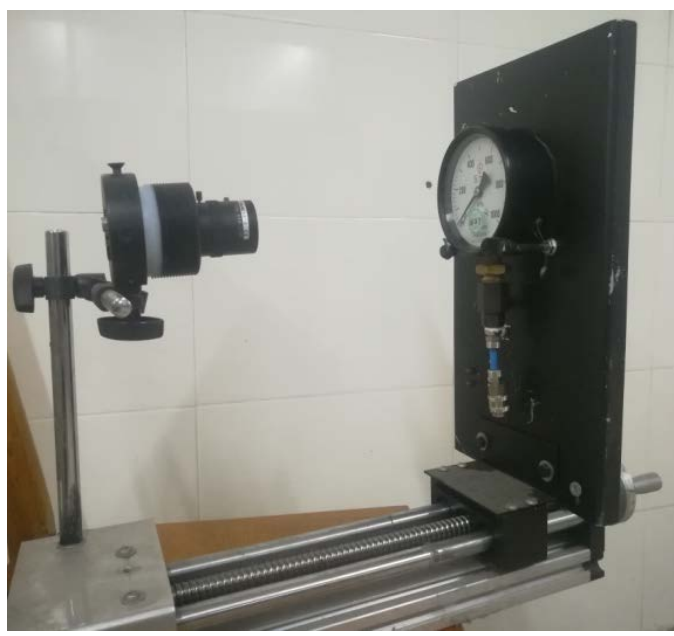

Figure 2. Test bench of tick-mark recognition. 


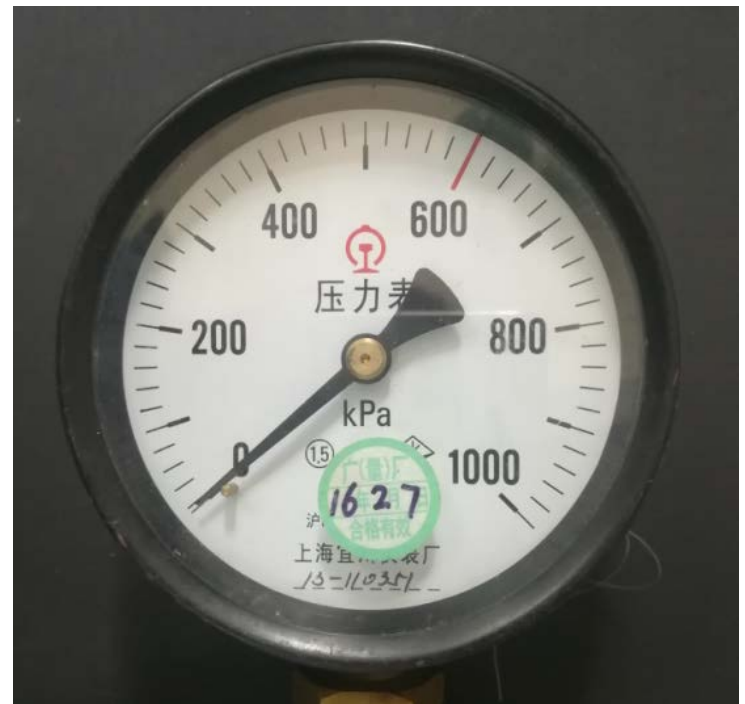

Figure 3. Image of analog pressure instrument.

1) Tick-mark area is uniformities in shape, size and brightness in image except for variant minority with characters which are bigger in shape.

2) Brightness value and contrast ratio of the tick-marks and their background take on stability to a certain extent.

3) Distances are almost equal between the outer border of the tick-marks and the instrument center.

4) Tick-marks lie symmetrically around the edge of upper instrument panel.

Following parameters are used to define the robustness of the previous algorithm quantitatively:

1) Employing $n_{\text {current,tick-mark }}$, the number of the pixels belonging to the

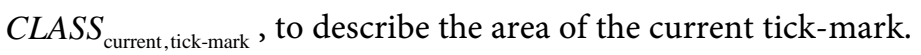

2) Employing $G A_{\text {current,tick-mark }}$, the brightness average of all pixels in tick-mark, to describe the brightness of the tick-mark, and employing $G A_{\text {current,background }}$, the brightness average of all pixels in tick-mark's background, to describe the brightness of tick-mark's background.

3) Employing $\Delta \theta_{\text {now }}$, the angle difference between the angle of the current tick-mark and its former tick-mark with reference to the instrument center, to describe the symmetry of the tick-marks.

In the procedure of the tick-mark searching with double image segmentation algorithm, if the adaptively changed parameters defined above including $n_{\text {current, tick-mark }}, G A_{\text {current, tick-mark }}, G A_{\text {current, background }}$ and $\Delta \theta_{\text {current }}$, take on strong quality of stability step by step, the searching procedure will be robust, and on the contrary, if the values of $n_{\text {current, tick-mark }}, G A_{\text {current, tick-mark }}, G A_{\text {current,background }}$ and $\Delta \theta_{\text {current }}$ sway sharply, the tick-mark searching algorithm will be susceptible to disturbance.

In addition, comparative tests were performed to verified the robustness of the tick-mark readout rate of DISA with Hough transformation, the classical line detection algorithm. 
Test 1: Basic function verification of DISA algorithm

Test conditions are listed as Table 4.

The values of the adaptively changed parameters, $n_{\text {current,tick-mark }}, G A_{\text {current,tick-mark }}$, $G A_{\text {current,background }}$ and $\Delta \theta_{\text {current }}$, are shown in Figures 3-6 and Table 5 respectively.

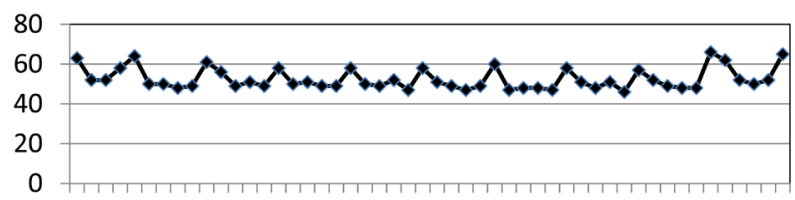

$\begin{array}{lllllllllllllllll}1 & 4 & 7 & 10 & 13 & 16 & 19 & 22 & 25 & 28 & 31 & 34 & 37 & 40 & 43 & 46 & 49\end{array}$

Figure 4. $n_{\text {current, tick-mark }}$, the number of the pixels in $C L A S S_{\text {current,tick-mark }}$ in each tick-mark.

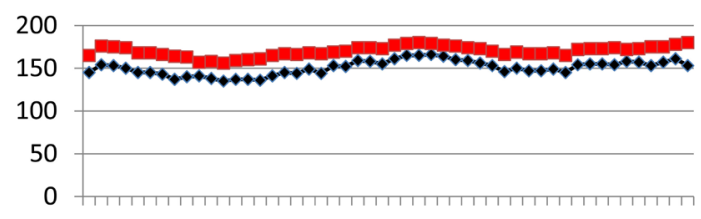

1471013161922252831343740434649

Figure 5. $G A_{\text {current,tick-mark }}$ (red points), $G A_{\text {current,background }}$ (blank points) the average grey of all pixels and its background in each tick-mark.

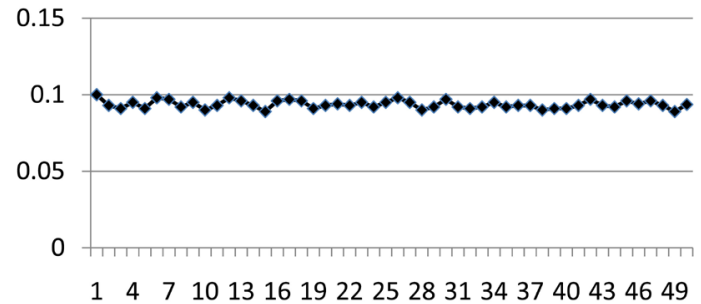

Figure 6. $\Delta \theta_{\text {current }}$, the angle difference between the angle of the current tick-mark and the previous tick-mark with reference to the instrument center.

Table 4. Test conditions.

\begin{tabular}{cc}
\hline time & Daytime at about 12:00 \\
weather & sunshine \\
site & indoor \\
illumination intensity & 1000 Lux approximately \\
sampled image & clear and tick-mark distinguishable with eye
\end{tabular}

Table 5. Variance, average and the their ratio for $n_{\text {current,tick-mark }}, G A_{\text {current,tick-mark }}$, $G A_{\text {current,background }}$ and $\Delta \theta_{\text {current }}$.

\begin{tabular}{ccccc}
\hline & $n_{\text {current, tick-mark }}$ & $G A_{\text {current, tick-mark }}$ & $G A_{\text {curren, background }}$ & $\Delta \theta_{\text {now }}$ \\
\hline Variance & 5.3 & 8.3 & 6.1 & 0.003 \\
average & 52.2 & 150.1 & 169.9 & 0.094 \\
variancel average & $10.1 \%$ & $5.5 \%$ & $3.6 \%$ & $3.2 \%$ \\
\hline
\end{tabular}


As shown in Figure 4, horizontal ordinate is the index of each tick-mark, and longitudinal coordinate is $n_{\text {current,tick-mark }}$, the number of the pixels in each tickmark in CLASS $S_{\text {current, tick-mark }}$.

From the data in Figure 4, it is observed that $n_{\text {current,tick-mark }}$ retains stable to some extent in searching procedure with periodic fluctuation ranging from 46 to 65 in every 5 tick-marks and the indexes of the peak points are 1, 5, 10, 15, 20, $25,30,35,40,45$ and 50. Furthermore, observation of tested instrument panel in Figure 3 shows that there are as many as 11 big tick-marks whose indexes, same as the peak points in Figure 4, are 1, 5, 10, 15, 20, 25, 30, 35, 40, 45 and 50 too. With data comparison in Figure 3 and Figure 4, conclusion can be easily drawn that the big tick-marks have more tick-mark pixels than the others, and the other small tick marks, though smaller in number, retain stable in number of pixels and distinguishable.

As shown in Figure 5, horizontal ordinate is the index of each tick-mark, and longitudinal coordinate are $G A_{\text {current,tick-mark }}, G A_{\text {current,background }}$, the average grey of all pixels and its background in each tick-mark in corresponding with the black and red curve respectively.

From the data in Figure 5, it is observed that $G A_{\text {current,background }}$ and $G A_{\text {current,tick-mark }}$ retains stable to some extent in searching procedure and the former is bigger than the latter at each tick-mark. In addition, the difference between them is no less than 20 which makes it possible to draw a line between each tick-mark and its background in grey value. Further, both $G A_{\text {current,background }}$ and $G A_{\text {current, tick-mark }}$ show same fluctuation with big-small-big-small-big, the reason for which is the uneven distribution of the light on the instrument panel.

As shown in Figure 6, horizontal ordinate is the index of each tick-mark, and longitudinal coordinate $\Delta \theta_{\text {current }}$, is the angle difference between the angle of the current tick-mark and the previous tick-mark with reference to the instrument center.

From the data in Figure 6, it is observed that the values of $\Delta \theta_{\text {current }}$, lie in the range from $0.89 \mathrm{rad}-0.1 \mathrm{rad}$ stably, and the variance of $\Delta \theta_{\text {current }}$, is no more than $0.005 \mathrm{rad}$. These data add weight to the evidence with accuracy and robustness of the algorithm.

As shown in Table 5, the ratios of variance and average for $G A_{\text {current,tick-mark }}$, $G A_{\text {current,background }}, D_{\text {current }}$ and $\Delta \theta_{\text {current }}$ are all no more than 5.5\%, and these data give a strong evidence of DISA'S robustness. In addition, the ratios of variance and average for $n_{\text {now,scale }}$ is as high as $10.1 \%$, which is the real reflection of the big tick-marks.

Figure 7 shows the recognized tick-marks colored with red and labeled with green cross, and the green cross in the center is the label of the estimated instrument center (Figure 8).

Summary: Test data shows that tick-marks on the pressure instrument can be recognized with no omission on condition of clear and tick-mark distinguishable image, and the parameters under investigation show robustly stable. 


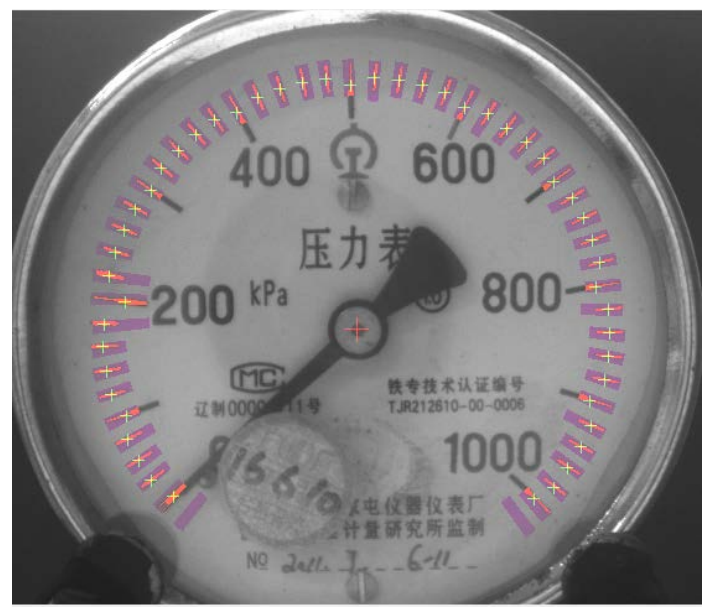

Figure 7. Recognized tick-marks colored with red and labeled with green cross.

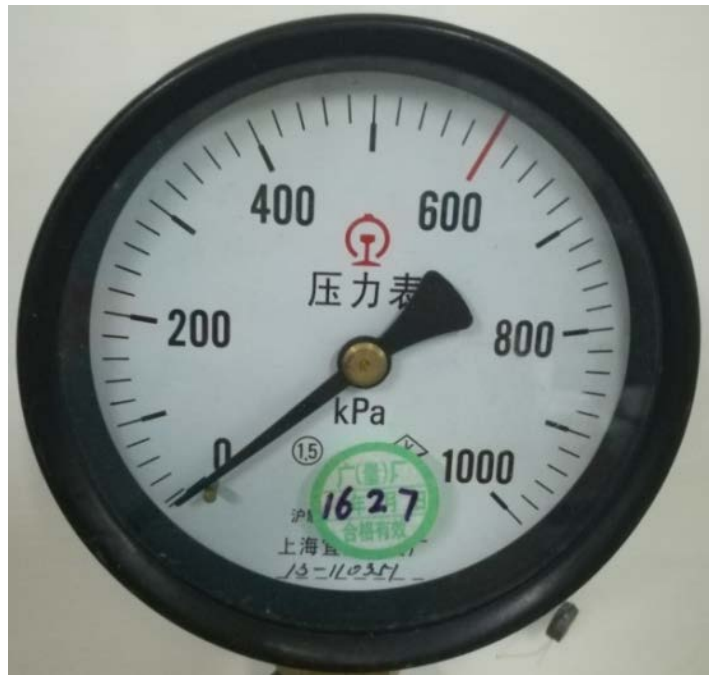

Figure 8. Panel of pressure instrument with abnormal tick-mark (red).

Test 2: Comparison tests with Hough Line Detection

Hough Transformation, a classical algorithm for line detection, has been employed to search tick marks in analog instrument recognition in [14]. In order to evaluate DISA, test conditions are designed to examine the tick-mark recognizing capacity of DISA in comparison with Hough Line Detection as shown in Table 6 and Table 7 in addition with 4 kinds of analog instruments shown in Figures 9-12.

Test is repeated 5 times for each panel and each illumination intensity, and 100 times tests are run totally with two algorithms above and results are shown in Table 9 with DISA and Table 8 with Hough Line Detection.

As shown in Table 8 and Table 9, for air packed instruments, type 0 , type 1 and type 3 , the tick-marks were $100 \%$ successfully recognized with the two algorithms above on condition of illumination intensity ranging from $2000 \mathrm{~lx}$ to $2500 \mathrm{~lx}$ with 30 times of reading, however, if the illumination intensity attenuate to no brighter than $1500 \mathrm{~lx}$, there happened to be 7 times and 11 times of failure 


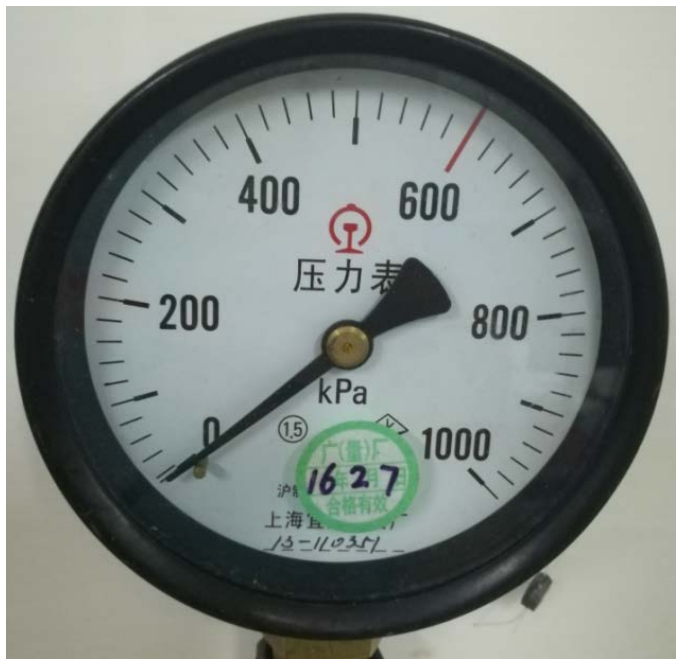

Figure 9. Type 0.

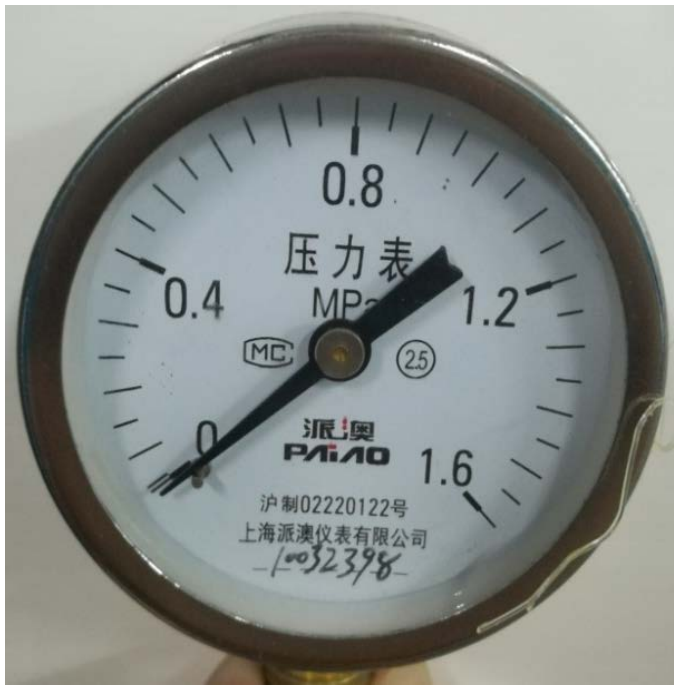

Figure 10. Type 1.

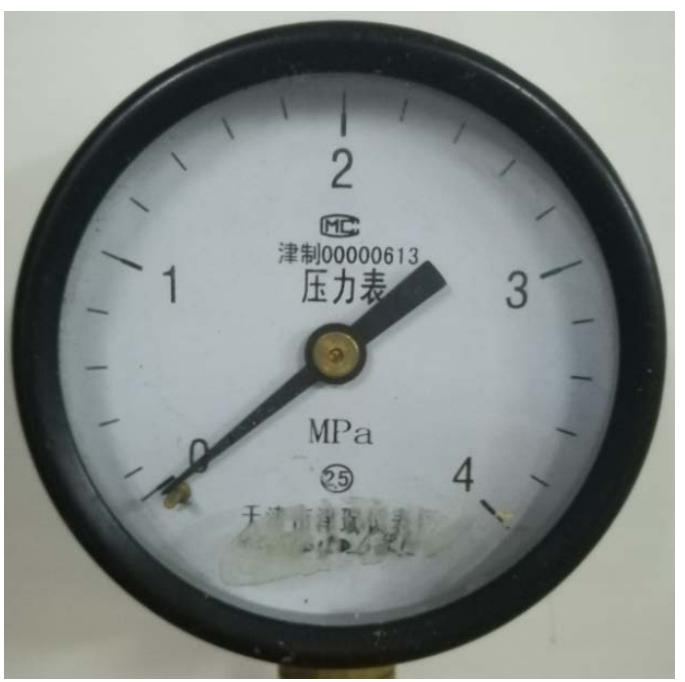

Figure 11. Type 2. 


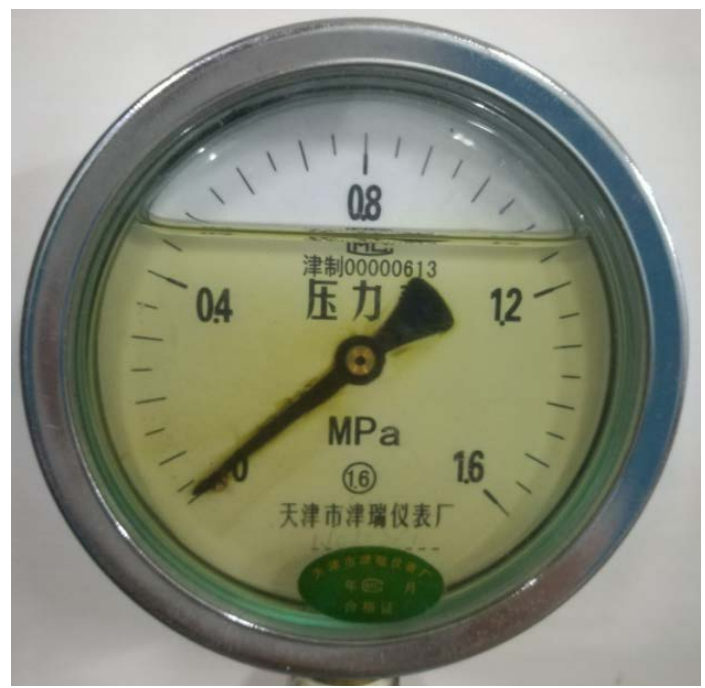

Figure 12. Type 3.

Table 6. 4 kinds of selected instruments.

\begin{tabular}{cccc}
\hline & panel diameter $(\mathrm{mm})$ & number of valid tick-marks & packing medium \\
\hline type 0 & 100 & 50 & air \\
type 1 & 100 & 50 & air \\
type 2 & 40 & 32 & air \\
type 3 & 40 & 20 & oil \\
\hline
\end{tabular}

Table 7. Illumination intensity conditions.

\begin{tabular}{ccccc}
\hline $\begin{array}{c}\text { illumination } \\
\text { intensity 0 }\end{array}$ & $\begin{array}{c}\text { illumination } \\
\text { intensity } 1\end{array}$ & $\begin{array}{c}\text { illumination } \\
\text { intensity } 2\end{array}$ & $\begin{array}{c}\text { illumination } \\
\text { intensity } 3\end{array}$ & $\begin{array}{c}\text { illumination } \\
\text { intensity } 4\end{array}$ \\
\hline $2500 \pm 250 \mathrm{~lx}$ & $2000 \pm 200 \mathrm{~lx}$ & $1500 \pm 150 \mathrm{~lx}$ & $1000 \pm 100 \mathrm{~lx}$ & $500 \pm 50 \mathrm{~lx}$ \\
\hline
\end{tabular}

Table 8. Test results of DISA.

\begin{tabular}{ccccc}
\hline TOVR & Type 0 & Type 1 & Type 2 & Type 3 \\
\hline illumination intensity 0 & 5 & 5 & 5 & 4 \\
illumination intensity 1 & 5 & 5 & 5 & 4 \\
illumination intensity 2 & 5 & 4 & 5 & 3 \\
illumination intensity 3 & 5 & 4 & 4 & 3 \\
illumination intensity 4 & 4 & 3 & 4 & 2 \\
\hline
\end{tabular}

Note: Times of valid recognition is abbreviated as TOVR.

Table 9. Test results of Hough line detection.

\begin{tabular}{ccccc}
\hline TOVR & Type 0 & Type 1 & Type 2 & Type 3 \\
\hline illumination intensity 0 & 5 & 5 & 5 & 0 \\
illumination intensity 1 & 5 & 5 & 5 & 0 \\
illumination intensity 2 & 5 & 4 & 3 & 0 \\
illumination intensity 3 & 4 & 3 & 3 & 0 \\
illumination intensity 4 & 4 & 3 & 3 & 0 \\
\hline
\end{tabular}


recognitions out of 45 times of tests respectively for DISA and for Hough Line Detection. For oil packed instrument, type 3, DISA had 9 times of failure recognitions out of 25 times of tests, whereas Hough Line Detection got no success record at all.

Panel image of type 1 instrument is under deep investigation to find the reason for failure recognition. As shown in Figure 12, type 3 instrument is packed with oil and there is a clear air-oil boundary in upper half of the instrument panel, and there exists evident grey difference between air zone and the oil zone. This brings difficulty to tick-mark recognition because of the uneven grey in the bright air zone and in the gray oil zone, and what's worse, there are many significant similarities between the air-oil boundary and the tick marks such as linear structure, brightness contrast with their backgrounds, dimension in width which lead to confusion in recognizing. Data in Table 9 show $100 \%$ recognition of type 3 instrument on illumination intensity from $2000 \mathrm{~lx}$ to $2500 \mathrm{~lx}$, and $73 \%$ recognition when illumination intensity is lower than $1500 \mathrm{~lx}$. On the contrary, data in Table 9 show total recognition failure for type 1 with Hough Line Detection due to the air-oil boundary.

Summary: Batch tests are run at 5 kinds of illumination intensity with 4 kinds of analog pressure instruments. As shown in test data, for the air packed instruments, DISA and Hough Line Detection experience $91 \%$ and $85 \%$ successful tick mark recognition in total respectively. In contrast with the oil packed instrument, influenced by an air-oil boundary, DISA only retains $64 \%$ successful recognition records, and Hough Line Detection had none.

\section{Conclusions}

Many factors such as the generally stained surface of pointer meter, and/or reflection and refraction of light would lead to shortage of the accuracy and robustness in tick-mark reading. Double image segmentation algorithm (DISA) is employed to solve this problem which, on the one hand, coarsely searches the tick-mark searching pitch one by one depending on the parameters including center coordinate of the instrument, distance between instrument center and estimated tick-mark center and the estimated length of tick-mark, and on the other hand, accurately picks up tick-mark from background depending on the estimated thresholds of tick-mark and background. Tests have been conducted with $640 * 480$ pixel industry camera and 4 kinds of pointer pressure meter, and the results show that read-out rate of tick-mark in pointer meter reached $100 \%$ at sufficient illumination and $76 \%$ at insufficient illumination for air packed instrument. In addition with oil packed instrument, DISA still experienced $64 \%$ recognition in comparison with 0 success record by Hough transformation.

Enhancement of tick mark recognition in harsh environment is an important target for industrial application, and more attention should be focused on following problems in future research:

1) Some tick marks in shadow and others in light arising from oblique light. 
2) Blur image leading to small grey difference between tick marks and their background.

3) Heavily stained surface leading to shade of part tick marks.

\section{Acknowledgements}

Financial support by the Technology Research Plan of Guangzhou under contract 201804010134 and Technology Research Plan of Guangdong Baiyun University under contract BYKY2016Z1 is gratefully appreciated. Many thanks go to professor Chunjing Lin (Guangdong Baiyun University) for his help and controller implementation for the testbench and also to Wei Xie (South China University of Technology) for all the helpful discussions.

\section{Conflicts of Interest}

The authors declare no conflicts of interest regarding the publication of this paper.

\section{References}

[1] Corrêa Alegria, F. and Cruz Serra, A. (2000) Computer Vision Applied to the Automatic Calibration of Measuring Instruments. Measurement, 28, 185-195. https://doi.org/10.1016/S0263-2241(00)00011-7

[2] Zheng, C., Wang, S.R., Zhang, Y.H., Zhang, P.X. and Zhao, Y. (2016) A Robust and Automatic Recognition System of Analog Instruments in Power System by Using Computer Vision. Measurement, 92, 413-420. https://doi.org/10.1016/j.measurement.2016.06.045

[3] Wang, B., Chen, L.L. and Cheng, J. (2018) New Result on Maximum Entropy Threshold Image Segmentation Based on P System. Optik, 163, 81-85. https://doi.org/10.1016/j.ijleo.2018.02.062

[4] Mittal, H. and Saraswat, M. (2018) An Optimum Multi-Level Image Thresholding Segmentation Using Non-Local Means 2D Histogram and Exponential Kbest Gravitational Search Algorithm. Engineering Applications of Artificial Intelligence, 71, 226-235. https://doi.org/10.1016/j.engappai.2018.03.001

[5] Chondro, P., Yao, C.-Y., Ruan, S.-J. and Chien, L.-C. (2018) Low Order Adaptive Region Growing for Lung Segmentation on Plain Chest Radiographs. Neurocomputing, 275, 1002-1011. https://doi.org/10.1016/j.neucom.2017.09.053

[6] Parida, P. and Bhoi, N. (2017) Wavelet Based Transition Region Extraction for Image Segmentation. Future Computing and Informatics Journal, 2, 65-78. https://doi.org/10.1016/j.fcij.2017.10.005

[7] Biswas, S. and Hazra, R. (2018) Robust Edge Detection Based on Modified MooreNeighbor. Optik, 168, 931-943. https://doi.org/10.1016/j.ijleo.2018.05.011

[8] Gupta, D. and Anand, R.S. (2017) A Hybrid Edge-Based Segmentation Approach for Ultrasound Medical Images. Biomedical Signal Processing and Control, 31, 116 126. https://doi.org/10.1016/j.bspc.2016.06.012

[9] Otsu, N. (1979) A Threshold Selection Method from Gray Level Histograms. IEEE Transactions on Systems, Man, and Cybernetics, 9, 62-66. https://doi.org/10.1109/TSMC.1979.4310076

[10] Malarvel, M., Sethumadhavan, G., Bhagi, P.C.R., Kar, S. and Thangavel, S. (2017) 
An Improved Version of Otsu's Method for Segmentation of Weld Defects on X-Radiography Images. Optik-International Journal for Light and Electron Optics, 142, 109-118. https://doi.org/10.1016/j.ijleo.2017.05.066

[11] Goh, T.Y., Basah, S.N., Yazid, H., Safar, M.J.A. and Saad, F.S.A. (2018) Performance Analysis of Image Thresholding: Otsu Technique. Measurement, 114, 298-307. https://doi.org/10.1016/j.measurement.2017.09.052

[12] Chung, K.-L., Huang, Y.-H. and Tsai, S.-R. (2014) Orientation-Based Discrete Hough Transform for Line Detection with Low Computational Complexity. Applied Mathematics and Computation, 237, 430-437. https://doi.org/10.1016/j.amc.2014.03.128

[13] Mochizuki, Y., Torii, A. and Imiya, A. (2009) N-Point Hough Transform for Line Detection. Journal of Visual Communication and Image Representation, 20, 242253. https://doi.org/10.1016/j.jvcir.2009.01.004

[14] Wang, J.J., Tian, G.H., et al. (2017) An Automatic Reading Method of Pointer Instruments. Conference. 2017 Chinese Automation Congress (CAC), Jinan, 20-22 October 2017, 1448-1453. https://doi.org/10.1109/CAC.2017.8242995 